\title{
Identification of Parasite Presence on Thin Blood Splotch Images
}

\author{
IJCRR \\ Section: Healthcare \\ Sci. Journal Impact \\ Factor: $6.1(2018)$ \\ ICV: 90.90 (2018) \\ (c) (i) (8) \\ Copyright@IJCRR
}

\section{Venkata Naresh Mandhala ${ }^{1}$, Debnath Bhattacharyya ${ }^{1}$, D. Sushma ${ }^{2}$}

\author{
'Department of Computer Science and Engineering, Koneru Lakshmaiah Education Foundation, Vaddeswaram, Guntur, AP, India; \\ ${ }^{2}$ Department of Computer Science and Engineering, Vignan's Institute of Information Technology (A) Visakhapatnam 530049, AP, India.
}

\section{ABSTRACT}

Parasite is a bacterium that lives in a separate organism that functions as a host known as plasmodium. The parasite is vulnerable to malaria, dengue, typhoid diseases, etc. The presence of the parasite in blood smears can often lead to human death for some times. So, detecting and recognizing the parasite in blood splotch images at the early stages is very important to save human life.

Aim and Objective: The primary consideration in this article is to detect the parasite which occurs in red blood cells through blood splotch images in early stages in less time using a new image processing method.

Method: The method which is followed identifies the presence of parasite on blood smear images, was done in several steps. The first step of the method is to collect the input image from a laboratory taken through an electronic microscope. Then the image is further sent by converting the input image to the grayscale image using the standard method. Once the grayscale is obtained, the output image is further converted to the monochrome image. The pixel values of the image consist of only binary values using the "Otsu Threshold form." Then this monochrome image is converted to a matrix model and printed with the binary values.

Conclusion: The presence of parasites on the images will be displayed with the binary values by either one or zero on the output matrix model. Suppose the entire image is displayed as all zeros, In that case, it can be concluded to no parasite presence, and if any one's presence on the matrix model, then it can be observed that there is a presence of parasite on the blood smear blotch images.

Key Words: Parasite, Blood splotch images, Matrix, Binary values, Grayscale, Image Processing.

\section{INTRODUCTION}

Image processing is a method of carrying out such image operations to produce an enhanced image or collect user data. It is a signal processing type where pictures are entered, and the output can be images or characteristics. ${ }^{1}$ Today the processing of photographs is a technique that is rapidly increasing. It forms a central field of engineering and IT studies. For the processing of photographs, two methods are applied, namely analogue and digital imagery. Analogue picture processing can be used for hard copies, such as prints and photographs. ${ }^{2,3}$ The full details about an image and the parasite can be discussed in detail as subsections below.

\section{Image definition}

The image is an arrangement of pixels organized in columns and lines of a given width and height. The pixel value of every image is used. ${ }^{3,45}$ The following are various styles of images,

a. Binary image: Binary image is termed as a monochrome image. In this image, there are just two black and white values. Black is the value of a pixel 0 , and white is a pixel 1 .

b. Grayscale image: Grayscale is a spectrum from black to white monochromatic colours with 255 pixels. The combined shades of black and grey are present in this greyscale picture. Therefore, a grey picture just has white with no colour and grey variations. ${ }^{6,7,8}$

\section{Corresponding Author:}

Venkata Naresh Mandhala, Department of Computer Science and Engineering, Koneru Lakshmaiah Education Foundation, Vaddeswaram, Guntur, AP, India; Email: mvnaresh.mca@gmail.com

ISSN: 2231-2196 (Print) ISSN: 0975-5241 (Online)

Received: 05.07.2020

Revised: 08.08 .2020

Accepted: 10.09 .2020

Published: 06.10 .2020 
c. True-colour image: True colour is a colour standard of every 24-bit monitor line allowing the colours up to $16,777,216$ to be available., ${ }^{9,10}$

\section{Parasite}

The parasite is a bacterium that lives in a separate organism that functions as a host known as plasmodium, which acts as a host. The parasite is vulnerable to malaria, dengue, typhoid diseases, etc. The presence of parasites in blood smears can often lead to human death for some times. A plant or a group of animals that lives in or on someone else and feeds on that organism known as a parasite. To sustain its life cycle, the parasite uses its nutrients. ${ }^{5}$. It uses the power of the host to maintain itself. Infections caused by protozoa, helminth, or arthropods contain parasitic diseases. A parasite cannot live, develop, and increase without a host. That is why it never kills the host, but rarely is it possible, however pathogens can spread, and some can be fatal. There are a wide variety of parasites. Symptoms of the parasite that present in red blood cells are digestive problems, diarrhoea, skin issues, hives, itching, fever, constant hunger, Muscle and joint pains, etc. ${ }^{6,22}$ Therefore, we need to analyze and observe the symptoms at the starting stage and immediately refer a doctor to control the damage that might happen to a person.

\section{LITERATURE SURVEY}

Several authors had considered the identification of content on the images with several techniques. Some authors had given about the image processing techniques to be used, whereas some authors discussed the disease identification methods. The articles related to this problem had discussed as,

Bagri Neelima et al. presented the full details about the images and their presentations and given various techniques and methods that can be used to identify the content on the images. ${ }^{1}$ They had given a comparative study on these all techniques for a better understanding of the readers. Liu Suolan et.al.discussed the images, types of images and also discussed the encryption algorithms available for the researchers to work on images. ${ }^{2}$ Al-Obaisi Fida' et.al. had discussed the images and the content identification mechanisms on images in detail. They had given the method of identifying the content on images by the thermal imaging process. ${ }^{3}$

P.A. Pattanaik et.al. introduced a system for "Object detection technique for malaria parasite in thin blood smear images." This paper analyses malaria larvae. ${ }^{5}$ The contaminated red blood cell pixel count in thin-blood smears plays a critical function. This paper describes three steps of the computer vision target detection process, utilizing the kernel-based detection and the Kalman filtering method for malaria detection. Using kernel detection, which contains accurate pixel information, the proposed procedure enables the target of malaria infection to be accurately detected and located in excellent bloodstream images. Puwar Yashasvi et.al. proposed a system, "Automated and unsupervised detection of malarial parasites in microscopic images." More than 500 photographs from two separate laboratories are evaluated for the image-based technique. The objective is to differentiate between positive and negative malaria cases using thin smear images. Because of the unmonitored nature of the method, minimal human intervention is necessary to speed up the entire diagnostic process. The total malaria immunity is $100 \%$, and the specificity for all malaria parasite organisms varies in the range between 50 and $88 \%$. A Rehman et.al. proposed "Microscopic Malaria Parasitaemia Diagnosis and Grading on Benchmark Datasets." The detection and classification of malaria parasitemia are useful, although far from ideal. Incorrect diagnosis and rating caused enormous death rates in young children worldwide. The latest research examines the diagnosis and degree of automated malaria parasitemia by image analyses and computational vision techniques in thin blood streak digital images. The latest findings indicate that current approaches are partially or morphologically reliant on solutions to the computer-related vision-based microscopic malaria parasitemia diagnosis issue.

Ahmed and Mubarak Bashir et al., who introduced a system for "Detection of Malaria Parasites Using Digital Image Processing." to detect malaria parasites is infected or not infected based on erythrocyte samples' features. "Computer-Aided System for Red Blood Cell Classification in Blood Smear Image", proposed by the authors, namely Tomari Razali et al., Otsu levels, for instance, are used to remove the history of erythrocyte images and filters to minimize noise and unwanted gaps and also to determine whether red blood cell images are regular or irregular. "Automatic System for Classification of Erythrocytes Infected with Malaria and Identification of Parasites Life Stage"10 by the authors Savkarea et al., used the darkened photos for diagnosis, the RBC segmentation methods, and the overlapping cells, the watershed algorithm, and the methods or system for classification of erythrocytes that were contaminated and for the identification of life stages of a parasite. Bhattacharyya Debnath et al. had discussed the identification of cyst presence on MRI/CT scan images in the human brain. They had discussed the method of matrix generation using the monochrome images. ${ }^{11}$ However, they had used to remove the noise from the input image first, and then the Gaussian filter had sharpened the edges of the input images. ${ }^{12,13,14}$ Then the image is converted to grayscale images and then to the monochrome images, and the final matrix was generated..$^{15,16}$

\section{Observations from previous work}

After observing several works carried out by various authors, the current method was not used by any authors to identify 
the presence of parasite on blood splotch images. ${ }^{17,18}$ The previous authors did not use a matrix approach to combine monochrome images to classify the parasite presence with blood splotch images in the red blood cells from the different papers listed above. They also did not use any standard method to convert the input images to grayscale images to identify the parasite on blood splotch images. ${ }^{19,20,21}$ Hence, an attempt was made to take these findings into account. A new monochrome approach for the picture matrix with a new algorithm was employed to decide whether parasites are present in red blood cells of blood splotch scenarios, and results were discussed in detail in the results section.

\section{PROPOSED WORK}

The new algorithm that recognizes the parasite within the red blood cells and the algorithm can be observed as follows. It was taken into consideration in the current research to identify the parasite in the RBCs via blood splotch pictures.

\section{Process followed to identify parasite}

The full process of the current method used to identify the presence of parasites on the blood smear images can be explained in detail in stepwise.

1. First, the colour image of blood splotch images is taken as the input to identify the parasite.

2. The input picture is transformed into the Grayscale picture using the "Average form" for the second step of pre-processing.

$\mathrm{AVG}=(\mathrm{R}+\mathrm{G}+\mathrm{B}) / 3$

From the above equation, $\mathrm{R}$ is Red pixel. $\mathrm{G}$ is a Green pixel. $\mathrm{B}$ is the Blue pixel.

3. When segmentation is done, the Grayscale image is regarded as input, and by "Otsu Threshold form," it is transformed into a monochrome picture.

4. Here monochrome image contains two values that are black and white. Black represents the background image, and white represents the foreground image.

This is implemented by using the "fixed threshold method."

$(5,5)<5=55(5,5)>5=1$

5. From the above equation, the Black pixel is indicated by 0 , and the White pixel is indicated by 1 . $\mathrm{T}$ is a threshold value.

6. Pixel magnitude is calculated for each pixel in the monochrome image, and then the pixels are represented in a matrix format and are displayed as output. Matrix contains 1's \& 0's.

7. The matrix is created from the monochrome image obtained at the segmentation point to define the parasite in blood splotch pictures more clearly.

8. Binary values that contain zero's and one's in the matrix. The presence of parasite indicates ones, and the absence of parasite indicates zeros.

\section{FLOW CHART}

The diagram allows us to understand the method of parasite recognition better, one can know the full process of how the current method is used to identify the parasites on the smear images in a step by step process. This step by step process is clearly explained with the help of the following figure 1.

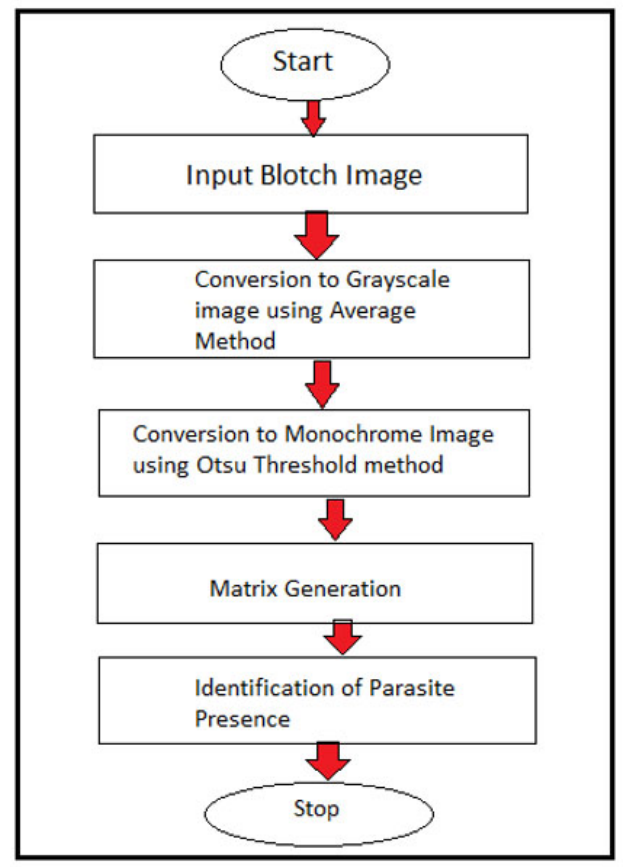

Figure 1: Flow Chart of the current proposed model for parasite identification.

The detailed process happening in the current model to identify the presence of parasites from the blood smear images process had explained in detail, and the full process can be observed in the above figure 1 in detail.

\section{RESULTS}

To better understand the problem and check the output of the current considered model, two cases were considered. In each case, a blood smear image has been collected from the laboratories, and the image had processed and tried to identify the presence of parasite on the image or not. The accuracy in identifying the presence of parasite on smear images will provide the developed model is either accurate or inaccurate. For checking the same, two images were considered, and the experimentation had done in two cases. The first case is about the image with parasite presence, and the second case is with the image without the presence of smear images. The two images were passed as input to the current model, and the output of the images was observed from the current model. The output of the current model will be a matrix form. The model is designed such that the output of the 
entire model will provide a matrix which is having the values binary values that is either one or zero. The entire picture is placed or printed in the form of a matrix with binary values. The location where the parasite is present will be displayed as one on the output matrix, and the location where no image or no traces of any parasite was found will be printed in the matrix as zero formats. Hence, the output of the entire model will be in the form of a matrix, and where the parasite is present, the output will be printed as 1 's in the matrix model and where there is no trace of any parasite, and then the matrix will be printed with all zeroes.

The details of the first case considered are,

Case Study 1: Identifying the parasite on the input image

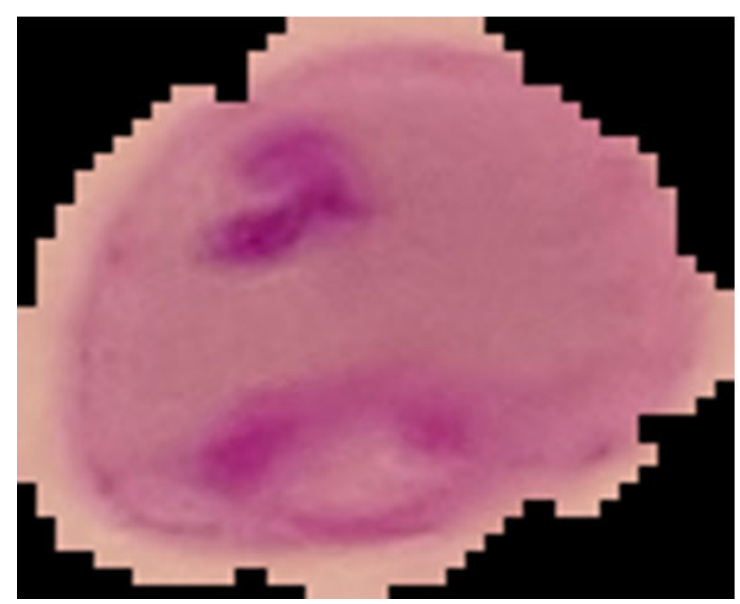

Figure 2: Input image of the first test case.

The sample input image or the splotch image was taken for testing in the current model can be observed in figure 2.

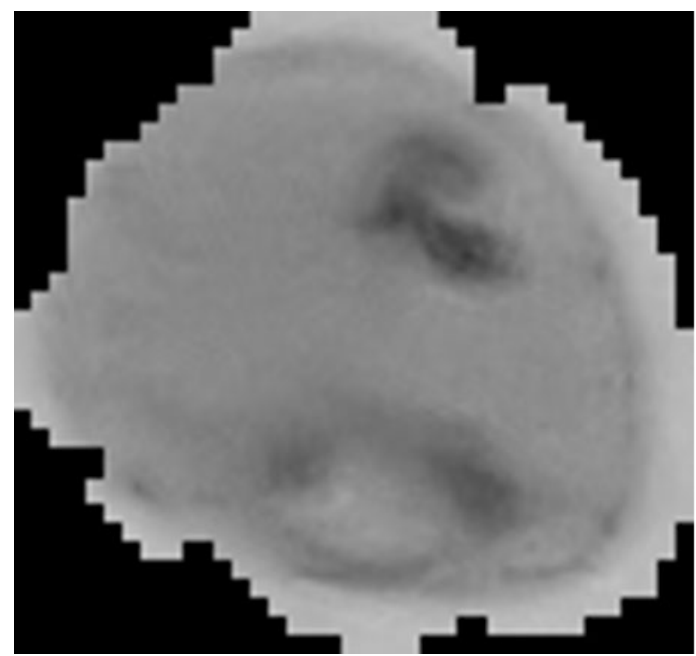

Figure 3: Gray Scale converted image for case 1.

Once the splotch image had been taken as input for the current model, the splotch image will be converted to the gray- scale image, and the converted grayscale image can be observed in figure 3.
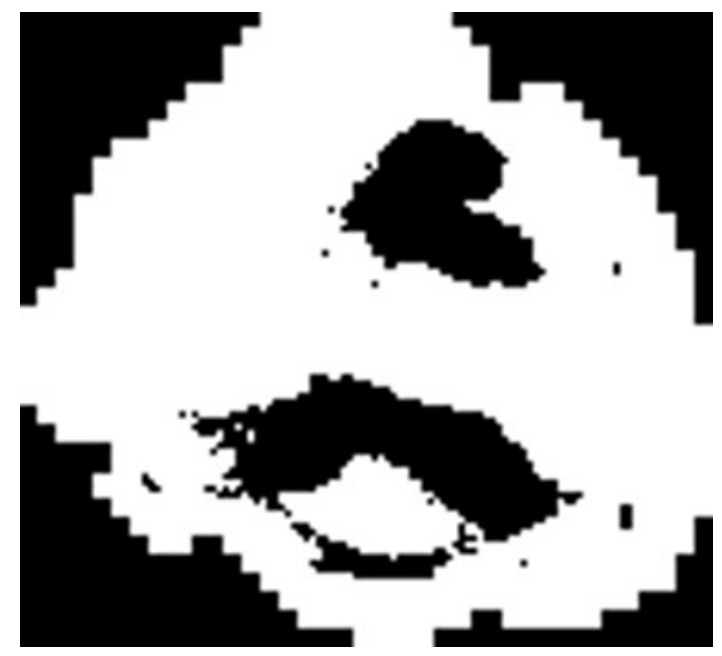

Figure 4: Monochrome Image obtained for case 1.

After converting from the splotch image to the grayscale image, the grayscale image will be converted further to the monochrome image. The obtained monochrome image can be observed in figure 4 .

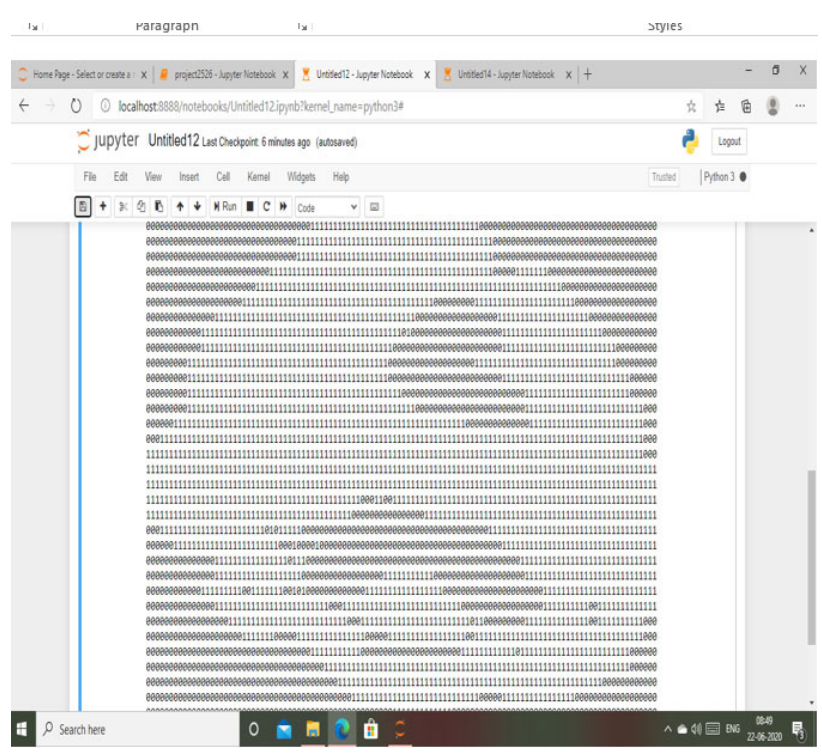

Figure 5: Matrix generated for case 1.

The matrix output image generated from the monochrome image can be observed in figure 5. From the above matrix, the 0 's represent the black pixels i.e., parasite presents in the red blood cell images, and 1's represents the white pixels. So, it shows that the input picture taken for testing gives an accurate result. Figure 5 shows that the current model is working well for the images with parasites on smear images.

The second case was the identification of parasite on the image with no parasite, the expected output of this model 
for this input image is all the values in the matrix should be printed with all zeroes as output matrix.

Case Study 2: Identifying the parasite in the input image

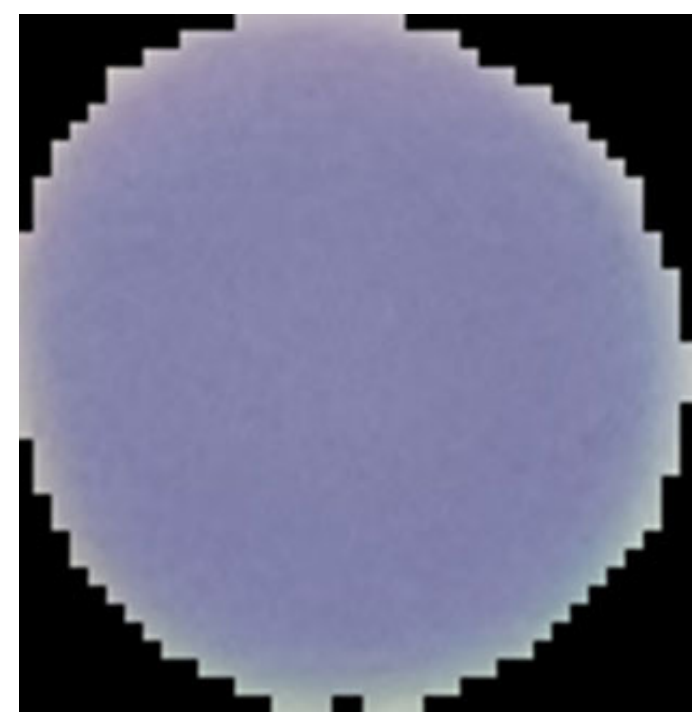

Figure 6: Input Image considered for case 2.

For further testing of the current model, another image as input had been considered, and this image can be sent to the model as the input image, and this splotch image can be observed in figure 6.

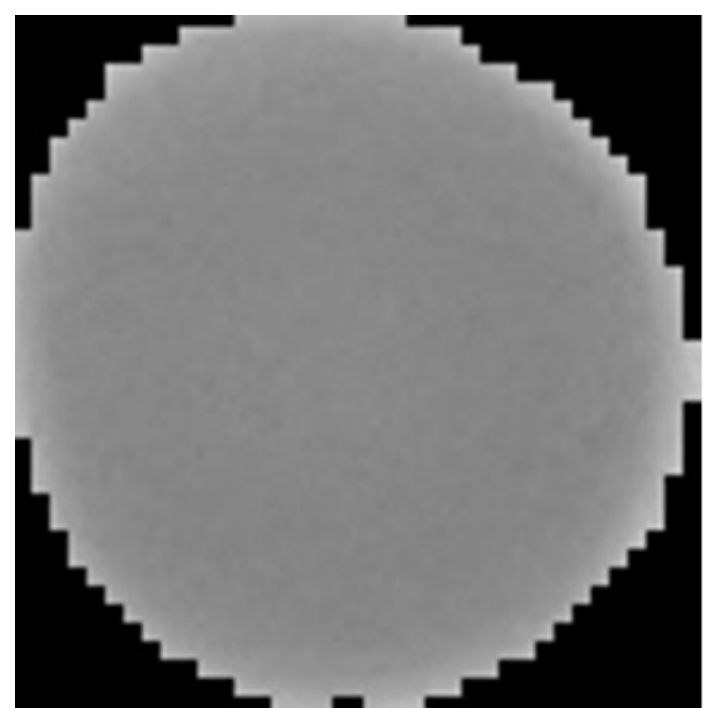

Figure 7: Grayscale Image obtained for the input image in case 2 .

The next phase of the conversion is to convert the splotch image to a grayscale image. The converted image can be observed in detail in figure 7.
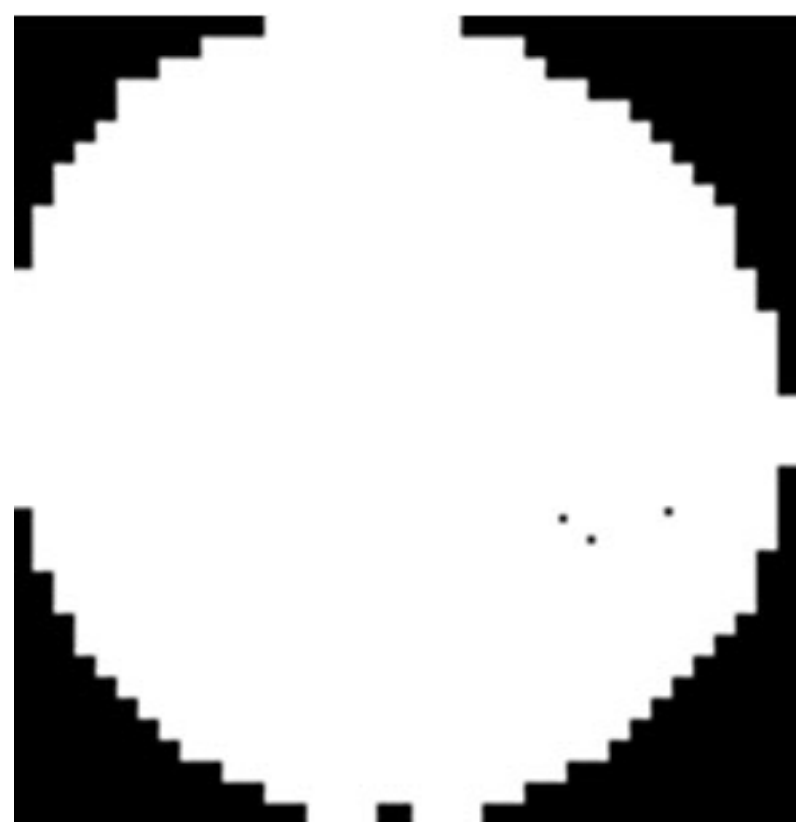

Figure 8: Monochrome Image for the input image in case 2.

After the conversion of the grayscale image, further, the grayscale image is converted to the monochrome image with binary values. The obtained monochrome image for the input splotch image can be observed in figure 8 .

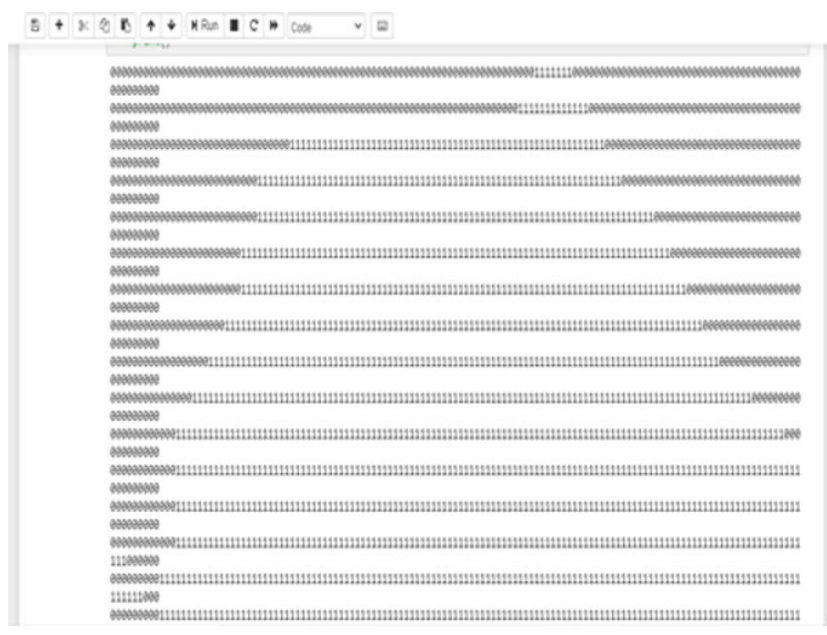

Figure 9: Matrix generated for the input image at case 2.

The output of the current model is a matrix with binary values, and this output matrix can be observed in figure 9. The above matrix shows only 1 's, so the parasite is not present in the input image, which is used for testing.

\section{Comparative study}

To understand the performance of the currently proposed method, a comparative analysis had been presented in comparison with the previously existing models for identifying the presence of a cyst in the human brain using MRI/CT scan images. 
Table I: Comparison of Current model results with the existing model results

\begin{tabular}{|c|c|c|c|c|c|}
\hline S. No. & $\begin{array}{l}\text { Types of Input } \\
\text { Images }\end{array}$ & Test Case & Input Image & $\begin{array}{l}\text { Existing Model } \\
\text { Detection Time }\end{array}$ & $\begin{array}{l}\text { Proposed Model } \\
\text { Detection Time }\end{array}$ \\
\hline 1 & $\begin{array}{l}\text { Thin Blood Smear } \\
\text { Images }\end{array}$ & $\begin{array}{l}\text { Absence of } \\
\text { Parasite }\end{array}$ & & 10 Seconds & 6 Seconds \\
\hline 2 & $\begin{array}{l}\text { Thin Blood Smear } \\
\text { Images }\end{array}$ & $\begin{array}{l}\text { Presence of } \\
\text { Parasite }\end{array}$ & & 12 Seconds & 5 Seconds \\
\hline 3 & $\begin{array}{l}\text { Thin Blood Smear } \\
\text { Images }\end{array}$ & $\begin{array}{l}\text { Presence of } \\
\text { Parasite }\end{array}$ & & 15 Seconds & 6 Seconds \\
\hline 4 & $\begin{array}{l}\text { Thin Blood Smear } \\
\text { Images }\end{array}$ & $\begin{array}{l}\text { Absence of } \\
\text { Parasite }\end{array}$ & & 11 Seconds & 5 Seconds \\
\hline
\end{tabular}

Table I observed that the currently considered model performs well compared to the previous model to identify the presence of parasite on the thin smear blood images.

\section{CONCLUSION}

The proposed system is capable of recognizing the presence of the parasite in red blood cell images in a slighter time using Image processing techniques. The parasite which occurs in blood cells changes the shape of blood cells. By considering this point, the matrix generated by a monochrome image that contains 0 's when the parasite presents in the image may lead to some diseases. So, automatic detection of the parasite using the "matrix method" results in slighter time while compared to the other segmentation methods like free threshold method and global threshold method.

Acknowledgement: Authors acknowledge the immense help received from the scholars whose articles are cited and included in references of this manuscript. The authors are also grateful to authors / editors / publishers of all those articles, journals and books from where the literature for this article has been reviewed and discussed.

\section{Conflict of Interest: Nil}

Source of Funding: Nil

\section{REFERENCES}

1. Bagri N, Johari PK. A comparative study on feature extraction using texture and shape for content-based image retrieval. International Journal of Advanced Science and Technology. 2015;80(4):41-52.

2. Liu S, Yue C, Wang H. An Improved Hybrid Encryption Algorithm for RGB Images. International Journal of Advanced Science and Technology. 2016 Oct 30;95:37-44.

3. Alqatawna JF, Faris H, Rodan A, Al-Kadi O. Pattern recognition of thermal images for monitoring of breathing function. International Journal of Control and Automation. 2015;8(6):381-92.

4. Kim K, Jeon J, Choi W, Kim P, Ho YS. Automatic cell classification in human's peripheral blood images based on morphological image processing. Australian Joint Conference on Artificial Intelligence 2001 Dec 10 (pp. 225-236). Springer, Berlin, Heidelberg.

5. Pattanaik PA, Swarnkar T, Sheet D. Object detection technique for malaria parasite in thin blood smear images. In2017 IEEE International Conference on Bioinformatics and Biomedicine (BIBM) 2017 Nov 13 (pp. 2120-2123). IEEE.

6. Purwar Y, Shah SL, Clarke G, Almugairi A, Muehlenbachs A. Automated and unsupervised detection of malarial parasites in 
microscopic images. Malaria journal. 2011 Dec 1;10(1):364.

7. Rehman A, Abbas N, Saba T, Mehmood Z, Mahmood T, Ahmed KT. Microscopic malaria parasitemia diagnosis and grading on benchmark datasets. Microscopy research and technique. 2018 Sep;81(9):1042-58.

8. Bashir A, Mustafa ZA, Abdelhamid I, Ibrahim R. Detection of malaria parasites using digital image processing. In2017 International Conference on Communication, Control, Computing and Electronics Engineering (ICCCCEE) 2017 Jan 16 (pp. 1-5). IEEE.

9. Tomari R, Zakaria WN, Jamil MM, Nor FM, Fuad NF. A computer-aided system for red blood cell classification in blood smear image. Procedia Computer Science. 2014 Jan 1;42:20613.

10. Savkare SS, Narote SP. Automatic system for classification of erythrocytes infected with malaria and identification of parasite's life stage. Procedia Technology. 2012 Jan 1;6:405-10.

11. Debnath Bhattacharyya, N.Thirupathi Rao. Detection of Cyst in Human Brain Using Scan Images. International Journal of Advanced Science and Technology. 2019;124:111-20.

12. Anitha, R., Jyothi, S., Mandhala, V. N., Bhattacharyya, D., Kim, T.-. Deep learning image processing technique for early detection of Alzheimer's disease. International Journal of Advanced Science and Technology. 2017;107:85-104.

13. Prasad, G. R. K., Siddaiah, N., Srinivas Babu, P. S. Design and model analysis of circular cantilever sensor for early detection of Parkinson's disease. Journal of Advanced Research in Dynamical and Control Systems. 2017;9(SP-16):433-44.

14. Pratuisha, K., Rajeswara Rao, D., Amudhavel, J., Murthy, J. V. R. A comprehensive study: On artificial-neural network techniques for estimation of coronary-artery disease. Journal of Advanced Research in Dynamical and Control Systems. 2017;9(SP-12):1673-83.
15. Razia, S., Narasingarao, M. R. A neuro computing frame work for thyroid disease diagnosis using machine learning techniques. Journal of Theoretical and Applied Information Technology. 2017;95(9):1996-05.

16. Tiruveedhula S., Narasingarao, M. R. Ensemble framework for classification of imbalanced malaria disease data. Journal of Advanced Research in Dynamical and Control Systems. 2017;9(SP-18):473-82.

17. Tiruveedhula S., Narasingarao, M. R. Machine learning techniques for malaria disease diagnosis - A review. Journal of Advanced Research in Dynamical and Control Systems. 2017;9(SP16):349-69.

18. Siva Kumar, P., Sarvani, V., Prudhvi Raj, P., Suma, K., Nandu, D. Prediction of heart disease using multiple regression analysis and support vector machines. Journal of Advanced Research in Dynamical and Control Systems. 2017;9(SP-18):675-682.

19. NagaGeetha M, Ramesh NV. An efficient IoT based smart irrigation system and plant diseases detection: A review. Int. J. of Eng. \& Technol. 2018;7(2.7):661-4.

20. Narasinga Rao, M. R., Sajana, T., Bhavana, N., Sai Ram, M., Nikhil Krishna, C. Prediction of chronic kidney disease using machine learning technique. Journal of Advanced Research in Dynamical and Control Systems. 2018;10:328-32.

21. Prasad GR, Shameem S, Srinivasulu N, Renukavalli C, Manasa B, Babu PS. Design and analysis of proof mass based microsensor for early detection of Parkinson's disease. International Journal of Engineering \& Technology. 2018;7(1.5):146-51.

22. Hemangi Joshi, Biren Parikh, Akash Thakkar, Manasvi Patel. Evaluation and Correlation of Abnormal Cell Flagging of Automated Haematology Analyzer with Peripheral Blood Film at a Hematology Laboratory in Tertiary Care Oncology Centre International Journal of Current Research and Review. 2020;12(12):20-25. 